\title{
A generalized Gronwall inequality and its application to fractional neutral evolution inclusions
}

\section{Zufeng Zhang ${ }^{1,2^{*}}$ and Zhangzhi Wei ${ }^{1,2}$}

\section{"Correspondence: jshyzzf@163.com 'Laboratory of Intelligent Information Processing, Suzhou University, Suzhou, Anhui 234000, P.R. China \\ ${ }^{2}$ School of Mathematics and Statistics, Suzhou University, Suzhou, Anhui 234000, P.R. China}

\begin{abstract}
This paper deals with the fractional neutral evolution differential inclusions. The existence results are established by using the fractional power of operators and a fixed point theorem for multivalued map. Moreover, we present a new generalized Gronwall inequality with singularity, which is an important tool in the proof of solvability.
\end{abstract}

MSC: $26 \mathrm{~A} 33 ; 34 \mathrm{~A} 60 ; 34 \mathrm{G} 25$

Keywords: generalized Gronwall inequality; fractional neutral evolution inclusions; mild solution; analytic semigroup

\section{Introduction}

In recent years, the theory of fractional differential equations is an important area of investigation for its wide applicability in the fields of physics, engineering, and economics. For more details, we refer to the monographs of Oldham and Spanier [1], Miller and Ross [2] and Podlubny [3] and papers [4-10].

This paper deals with the fractional neutral evolution inclusions of the form

$$
\begin{cases}D^{q}\left(x(t)-g\left(t, x_{t}\right)\right) \in-A x(t)+F\left(t, x_{t}\right), & t \in[0, b], \\ x(t)=\phi(t), & t \in[-\tau, 0],\end{cases}
$$

where $D^{q}$ represents the Caputo fractional derivative of order $0<q<1, b>0,-A$ is the infinitesimal generator of an analytic semigroup $\{T(t)\}_{t \geq 0}$ on a Banach space $X, F:[0, b] \times$ $C([-\tau, 0], X) \rightarrow \mathcal{P}(X)$ is a multivalued map with nonempty, bounded, convex, and closed values, $\mathcal{P}(X)$ is the family of all nonempty subsets of $X, g:[0, b] \times C([-\tau, 0], X) \rightarrow X$ is a given function to be introduced later, and $\phi \in C([-\tau, 0], X), \tau>0$. For any $x \in C([-\tau, b], X)$ and any $t \in[0, b]$, define $x_{t}(\varsigma)$ by $x_{t}(\varsigma)=x(t+\varsigma)$ for $\varsigma \in[-\tau, 0]$, where $x_{t}(\cdot)$ represents the history of the state from time $t-\tau$ up to the present time $t$.

Neutral fractional differential systems arise in many areas of applied mathematics and have received much attention recently. For some applications and recent results, we refer to [11-14] and the references therein. Very recently, Wang and Zhou [15] studied the existence and controllability results for fractional evolution differential inclusions involv-

(c) 2016 Zhang and Wei. This article is distributed under the terms of the Creative Commons Attribution 4.0 International License (http://creativecommons.org/licenses/by/4.0/), which permits unrestricted use, distribution, and reproduction in any medium, provided you give appropriate credit to the original author(s) and the source, provide a link to the Creative Commons license, and indicate if changes were made. 
ing the Caputo derivative in Banach spaces. Yan [16] investigated the controllability of fractional-order functional integro-differential inclusions with infinite delay in Banach spaces. We remark that the existence or the controllability results in these mentioned papers are obtained under strong assumptions on the nonlinear parts $f$ and $F$ in equations and inclusions, respectively. The purpose of this paper is to release the limitation on the nonlinear term. For this purpose, we first present a new generalized Gronwall inequality with singularity, which is effective in dealing with fractional neutral evolution systems.

The remainder of the paper is arranged as follows. In Section 2, we give some basic definitions and preliminary results. A new generalized Gronwall inequality is proved in Section 3. Section 4 is devoted to the existence result of mild solutions for problem (1.1). An example is presented in Section 5 to illustrate our main theorem.

\section{Preliminaries}

Let $(X,|\cdot|)$ be a Banach space, and $J \subset \mathbf{R}$. Then $C(J, X)$ is the Banach space consisting of all continuous functions from $J$ into $X$ equipped with the norm $\|y\|_{J}=\sup \{|y(t)|: t \in J\}$, and $B(X)$ denotes the Banach space of all bounded linear operators from $X$ into $X$ with the norm

$$
\|T\|=\sup \{|T(y)|:|y|=1\},
$$

where $T \in B(X)$ and $y \in X$.

For $1 \leq p \leq \infty$, define the norm of a measurable function $m: J \rightarrow \mathbf{R}$ by

$$
\|m\|_{L^{p} J}= \begin{cases}\left(\int_{J}|m(t)|^{p} d t\right)^{\frac{1}{p}}, & 1 \leq p<\infty \\ \inf _{\mu(\bar{J})=0}\left\{\sup _{t \in J-\bar{J}}|m(t)|\right\}, & p=\infty\end{cases}
$$

where $\mu(\bar{J})$ is the Lebesgue measure on $\bar{J}$. Denote by $L^{p}(J, \mathbf{R})$ the Banach space of all Lebesgue-measurable functions $m: J \rightarrow \mathbf{R}$ with the norm satisfying $\|\cdot\|_{L^{p_{J}}}<\infty$.

Lemma 2.1 (Hölder inequality) Let $r, p \geq 1$ and $\frac{1}{r}+\frac{1}{p}=1$. If $l \in L^{r}(J, \mathbf{R})$ and $m \in L^{p}(J, \mathbf{R})$, then $l m \in L^{1}(J, \mathbf{R})$ and $\|l m\|_{L^{1} J} \leq\|l\|_{L^{r} J}\|m\|_{L^{p} J}$.

Lemma 2.2 (Bochner theorem) A measurable function $x: J \rightarrow X$ is Bochner-integrable if $|x|$ is Lebesgue-integrable.

Let $-A$ be the infinitesimal generator of an analytic semigroup $\{T(t)\}_{t \geq 0}$ of uniformly bounded linear operators on $X$. Let $0 \in \rho(A)$, where $\rho(A)$ is the resolvent set of $A$. Then we can define the fractional power $A^{\alpha}$ for $0<\alpha \leq 1$ as a closed linear operator on the domain $D\left(A^{\alpha}\right)$. We have the following known results:

(i) There is $M \geq 1$ such that

$$
M:=\sup _{t \in[0,+\infty)}\|T(t)\| \leq \infty .
$$

(ii) For any $\alpha \in(0,1]$, there exists a positive constant $C_{\alpha}$ such that

$$
\left\|A^{\alpha} T(t)\right\| \leq \frac{C_{\alpha}}{t^{\alpha}}, \quad 0<t \leq b .
$$

For details on fractional powers of closed operators, we refer to [17]. 
Definition 2.3 ([3]) The Riemann-Liouville fractional integral of order $\alpha \in \mathbf{R}^{+}$of $f: \mathbf{R}^{+} \rightarrow$ $X$ is defined by

$$
I_{0}^{\alpha} f(t)=\frac{1}{\Gamma(\alpha)} \int_{0}^{t}(t-s)^{\alpha-1} f(s) d s, \quad t>0,
$$

where $\Gamma$ is the gamma function.

Definition 2.4 ([3]) The Caputo fractional derivative of order $0<\alpha<1$ of $f \in C^{1}([0, \infty)$, $X)$ is defined by

$$
D^{\alpha} f(t)=\frac{1}{\Gamma(1-\alpha)} \int_{0}^{t}(t-s)^{-\alpha} f^{\prime}(s) d s, \quad t>0
$$

Next, we present some basic definitions and results on multivalued maps. See [18] for more details.

Let $(X, d)$ be a metric space, and $\mathcal{P}(X)$ be the family for all nonempty subsets of $X$. We give the following notation:

$$
\begin{array}{ll}
\mathcal{P}_{c l}(X)=\{Y \in \mathcal{P}(X): Y \text { is closed }\}, & \mathcal{P}_{b d}(X)=\{Y \in \mathcal{P}(X): Y \text { is bounded }\}, \\
\mathcal{P}_{c v}(X)=\{Y \in \mathcal{P}(X): Y \text { is convex }\}, & \mathcal{P}_{c p}(X)=\{Y \in \mathcal{P}(X): Y \text { is compact }\} .
\end{array}
$$

A multivalued map $F: X \rightarrow \mathcal{P}(X)$ is convex (closed) valued if $F(x)$ is convex (closed) for all $x \in X ; F$ is bounded on bounded sets if $F(B)=U_{x \in B} F(x)$ is bounded in $X$ for all $B \in \mathcal{P}_{b d}(X)$, that is, $\sup _{x \in B}\{\sup \{|y|: y \in F(x)\}\}<\infty ; F$ is said to be upper semi-continuous (u.s.c. for short) on $X$ if for each $x_{0} \in X$, the set $F\left(x_{0}\right)$ is a nonempty, closed subset of $X$ and if for each open set $\mathcal{U}$ of $X$ containing $F\left(x_{0}\right)$, there is an open neighborhood $\mathcal{V}$ of $x_{0}$ such that $F(\mathcal{V}) \subset \mathcal{U}$; and $F$ is completely continuous if $F(B)$ is relatively compact for every $B \in \mathcal{P}_{b d}(X)$.

If a multivalued map $F$ is completely continuous and has nonempty compact values, then $F$ is u.s.c. if and only if $F$ has a closed graph, that is, $x_{n} \rightarrow x_{*}, y_{n} \rightarrow y_{*}, y_{n} \in F\left(x_{n}\right)$ imply $y_{*} \in F\left(x_{*}\right)$.

Consider $H_{d}: \mathcal{P}(X) \times \mathcal{P}(X) \rightarrow \mathbf{R}^{+} \cup\{\infty\}$ given by

$$
H_{d}(A, B)=\max \left\{\sup _{x \in A} d(x, B), \sup _{y \in B} d(A, y)\right\}
$$

where $d(A, y)=\inf _{x \in A} d(x, y), d(x, B)=\inf _{y \in B} d(x, y)$. Then $\left(\mathcal{P}_{b d, c l}(X), H_{d}\right)$ is a metric space, and $\left(\mathcal{P}_{c l}(X), H_{d}\right)$ is a generalized metric space (see [19]).

Definition 2.5 ([20]) A multivalued operator $\mathcal{N}: X \rightarrow \mathcal{P}_{c l}(X)$

(i) is a contraction if and only if there exists $0<\gamma<1$ such that, for each $x, y \in X$,

$$
H_{d}(\mathcal{N}(x), \mathcal{N}(y)) \leq \gamma d(x, y)
$$

(ii) has a fixed point if there exists $x \in X$ such that $x \in \mathcal{N}(x)$. 
Theorem 2.6 ([20]) Let J be a compact interval, and X be a Banach space. Let the map $F: J \times C(J, X) \rightarrow \mathcal{P}_{b d, c l, c v}(X),(t, u) \mapsto F(t, u)$, be measurable with respect to $t$ for each $u \in X$ and upper semicontinuous with respect to $u$ for each $t \in J$. Moreover, suppose that for each fixed $u \in C(J, X)$, the set

$$
\mathcal{N}_{F, u}=\left\{f \in L^{1}(J, X): f(t) \in F(t, u) \text { for a.e. } t \in J\right\}
$$

is nonempty. Also, let $\mathcal{T}$ be a linear continuous mapping from $L^{1}(J, X)$ to $C(J, X)$. Then the operator

$$
\mathcal{T} \circ \mathcal{N}_{F}: C(J, X) \rightarrow \mathcal{P}_{b d, c l, c v}(C(J, X)), \quad u \rightarrow\left(\mathcal{T} \circ \mathcal{N}_{F}\right)(u)=\mathcal{T}\left(\mathcal{N}_{F, u}\right)
$$

is a closed graph operator in $C(J, X) \times C(J, X)$.

The main tool in our approach is the following fixed point theorem.

Theorem 2.7 ([21, 22]) Let $X$ be a Banach space, and $\mathcal{A}: X \rightarrow \mathcal{P}_{c l, c v, b d}(X)$ and $\mathcal{B}: X \rightarrow$ $\mathcal{P}_{c p, c v}(X)$ be two multivalued operators satisfying

(a) $\mathcal{A}$ is a contraction, and

(b) $\mathcal{B}$ is upper semicontinuous and completely continuous.

Then, either

(i) the operator inclusion $\lambda x \in \mathcal{A} x+\mathcal{B} x$ has a solution for $\lambda=1$, or

(ii) the set $U=\{u \in X \mid u \in \lambda \mathcal{A} u+\lambda \mathcal{B} u, 0<\lambda<1\}$ is unbounded.

\section{A generalized Gronwall inequality}

In this section, we establish a generalized Gronwall inequality, which is important in proving the existence result. The proof is based on an iteration argument.

Lemma 3.1 ([23]) For $x \geq 1$, we have

$$
\left(\frac{x}{e}\right)^{x} \sqrt{2 \pi x}\left(1+\frac{1}{12 x}\right)<\Gamma(x+1)<\left(\frac{x}{e}\right)^{x} \sqrt{2 \pi x}\left(1+\frac{1}{12 x-0.5}\right) .
$$

Theorem 3.2 Suppose that $\alpha, \beta>0, a(t)$ is a nonnegative function locally integrable on $\mathbf{R}^{+}, g(t)$ and $h(t)$ are nonnegative, nondecreasing continuous functions defined on $\mathbf{R}^{+}$, and $u(t)$ is a nonnegative and locally integrable function on $\mathbf{R}^{+}$such that

$$
u(t) \leq a(t)+g(t) \int_{0}^{t}(t-s)^{\alpha-1} u(s) d s+h(t) \int_{0}^{t}(t-s)^{\beta-1} u(s) d s, \quad t \in \mathbf{R}^{+} .
$$

Then, for each constant $b \geq 0, t \in[0, b]$, we have

$$
u(t) \leq a(t)+\int_{0}^{t} \sum_{n=1}^{\infty} \sum_{k=0}^{n} C_{n}^{k} \frac{(g(t) \Gamma(\alpha))^{k}(h(t) \Gamma(\beta))^{n-k}}{\Gamma(k \alpha+(n-k) \beta)}(t-s)^{k \alpha+(n-k) \beta-1} a(s) d s .
$$

Proof Let $B \psi(t)=g(t) \int_{0}^{t}(t-s)^{\alpha-1} \psi(s) d s+h(t) \int_{0}^{t}(t-s)^{\beta-1} \psi(s) d s$ for locally integrable functions $\psi$. Then

$$
u(t) \leq a(t)+B u(t)
$$


By iteration we have

$$
u(t) \leq \sum_{k=0}^{n-1} B^{k} a(t)+B^{n} u(t)
$$

Next, we shall prove the following two statements:

(i) $B^{n} u(t) \leq \int_{0}^{t} \sum_{k=0}^{n} C_{n}^{k} \frac{(g(t) \Gamma(\alpha))^{k}(h(t) \Gamma(\beta))^{n-k}}{\Gamma(k \alpha+(n-k) \beta)}(t-s)^{k \alpha+(n-k) \beta-1} u(s) d s$.

(ii) For each $t \in[0, b], \lim _{n \rightarrow \infty} B^{n} u(t)=0$, and $\sum_{n=1}^{\infty} B^{n} a(t)$ is convergent.

Proof of (i). From the definition of $B$ we have (i) is true for $n=1$. Assume that (i) is true for some $n=m$. Then, for $n=m+1$,

$$
\begin{aligned}
B^{m+1} u(t)= & B\left(B^{m} u(t)\right) \\
= & g(t) \int_{0}^{t}(t-s)^{\alpha-1} B^{m} u(s) d s+h(t) \int_{0}^{t}(t-s)^{\beta-1} B^{m} u(s) d s \\
\leq & g(t) \int_{0}^{t}(t-s)^{\alpha-1} \int_{0}^{s} \sum_{k=0}^{m} C_{m}^{k} \frac{(g(t) \Gamma(\alpha))^{k}(h(t) \Gamma(\beta))^{m-k}}{\Gamma(k \alpha+(m-k) \beta)} \\
& \times\left(s-s_{1}\right)^{k \alpha+(m-k) \beta-1} u\left(s_{1}\right) d s_{1} d s \\
& +h(t) \int_{0}^{t}(t-s)^{\beta-1} \int_{0}^{s} \sum_{k=0}^{m} C_{m}^{k} \frac{(g(t) \Gamma(\alpha))^{k}(h(t) \Gamma(\beta))^{m-k}}{\Gamma(k \alpha+(m-k) \beta)} \\
& \times\left(s-s_{1}\right)^{k \alpha+(m-k) \beta-1} u\left(s_{1}\right) d s_{1} d s .
\end{aligned}
$$

By interchanging the order of integration and by the equality $C_{m}^{n}+C_{m}^{n+1}=C_{m+1}^{n+1}$ we have

$$
\begin{array}{rl}
B^{m+1} & u(t) \\
\leq & \int_{0}^{t} g(t) u\left(s_{1}\right) \int_{s_{1}}^{t} \sum_{k=0}^{m} C_{m}^{k} \frac{(g(t) \Gamma(\alpha))^{k}(h(t) \Gamma(\beta))^{m-k}}{\Gamma(k \alpha+(m-k) \beta)}(t-s)^{\alpha-1}\left(s-s_{1}\right)^{k \alpha+(m-k) \beta-1} d s d s_{1} \\
& +\int_{0}^{t} h(t) u\left(s_{1}\right) \int_{s_{1}}^{t} \sum_{k=0}^{m} C_{m}^{k} \frac{(g(t) \Gamma(\alpha))^{k}(h(t) \Gamma(\beta))^{m-k}}{\Gamma(k \alpha+(m-k) \beta)} \\
& \times(t-s)^{\beta-1}\left(s-s_{1}\right)^{k \alpha+(m-k) \beta-1} d s d s_{1} \\
= & \int_{0}^{t} \sum_{k=0}^{m} C_{m}^{k} \frac{(g(t) \Gamma(\alpha))^{k+1}(h(t) \Gamma(\beta))^{m-k}}{\Gamma((k+1) \alpha+(m-k) \beta)} u\left(s_{1}\right)\left(t-s_{1}\right)^{(k+1) \alpha+(m-k) \beta-1} d s_{1} \\
& +\int_{0}^{t} \sum_{k=0}^{m} C_{m}^{k} \frac{(g(t) \Gamma(\alpha))^{k}(h(t) \Gamma(\beta))^{m+1-k}}{\Gamma(k \alpha+(m+1-k) \beta)} u\left(s_{1}\right)\left(t-s_{1}\right)^{k \alpha+(m+1-k) \beta-1} d s_{1} \\
= & \int_{0}^{t} \sum_{k=0}^{m+1} C_{m+1}^{k} \frac{(g(t) \Gamma(\alpha))^{k}(h(t) \Gamma(\beta))^{m-k}}{\Gamma(k \alpha+(m-k) \beta)} u\left(s_{1}\right)\left(t-s_{1}\right)^{k \alpha+(m-k) \beta-1} d s_{1} .
\end{array}
$$

Therefore, by induction we get that (i) is true.

Proof of (ii). First, there exists $N_{1}>0$ such that for $n>N_{1}$, we have

$$
k \alpha+(n-k) \beta-1>0 \quad \text { for } k=0,1,2, \ldots, n .
$$


Take arbitrary $b>0$. Then, for $n>N_{1}, k=0,1, \ldots, n$, we have

$$
(t-s)^{k \alpha+(n-k) \beta-1} \leq b^{k \alpha+(n-k) \beta-1}, \quad t \in[0, b], s \in[0, t] .
$$

By Lemma 3.1, for $n>N_{1}$, we have

$$
\begin{aligned}
\sum_{k=0}^{n} & C_{n}^{k} \frac{(g(t) \Gamma(\alpha))^{k}(h(t) \Gamma(\beta))^{n-k}}{\Gamma(k \alpha+(n-k) \beta)} b^{k \alpha+(n-k) \beta-1} \\
\leq & \sum_{k=0}^{n} C_{n}^{k} \frac{(g(t) \Gamma(\alpha))^{k}(h(t) \Gamma(\beta))^{n-k}}{\left(\frac{k \alpha+(n-k) \beta-1}{e}\right)^{k \alpha+(n-k) \beta-1} \sqrt{2 \pi(k \alpha+(n-k) \beta-1)}} b^{k \alpha+(n-k) \beta-1} \\
\leq & \sum_{k=0}^{n} C_{n}^{k}\left[\frac{g(T) \Gamma(\alpha) b^{\alpha}}{\left(\frac{k \alpha+(n-k) \beta-1}{e}\right)^{\alpha}}\right]^{k}\left[\frac{h(T) \Gamma(\beta) b^{\beta}}{\left(\frac{k \alpha+(n-k) \beta-1}{e}\right)^{\beta}}\right]^{n-k} \\
& \times \frac{e}{b(k \alpha+(n-k) \beta-1) \sqrt{2 \pi(k \alpha+(n-k) \beta-1)}}
\end{aligned}
$$

Choosing constants $c_{1}, c_{2}>0$ satisfying $0<c_{1}+c_{2}<1$, we can easily get that there is an integer constant $N>N_{1}$ such that, for $n>N, k=0,1,2, \ldots, n$,

$$
\begin{aligned}
& \frac{g(T) \Gamma(\alpha) b^{\alpha}}{\left(\frac{k \alpha+(n-k) \beta-1}{e}\right)^{\alpha}}<c_{1}, \quad \frac{h(T) \Gamma(\beta) b^{\beta}}{\left(\frac{k \alpha+(n-k) \beta-1}{e}\right)^{\beta}}<c_{2}, \\
& \frac{e^{b(k \alpha+(n-k) \beta-1) \sqrt{2 \pi(k \alpha+(n-k) \beta-1)}}<1 .}{} \text {. }
\end{aligned}
$$

Therefore, for $n>N$ and $t \in[0, b]$, we have

$$
\begin{aligned}
\lim _{n \rightarrow \infty} B^{n} u(t) & \leq \lim _{n \rightarrow \infty} \int_{0}^{t} \sum_{k=0}^{n} C_{n}^{k} \frac{(g(t) \Gamma(\alpha))^{k}(h(t) \Gamma(\beta))^{n-k}}{\Gamma(k \alpha+(n-k) \beta)}(t-s)^{k \alpha+(n-k) \beta-1} u(s) d s \\
& \leq \lim _{n \rightarrow \infty} \int_{0}^{t} \sum_{k=0}^{n} C_{n}^{k} \frac{(g(t) \Gamma(\alpha))^{k}(h(t) \Gamma(\beta))^{n-k}}{\Gamma(k \alpha+(n-k) \beta)} b^{k \alpha+(n-k) \beta-1} u(s) d s \\
& \leq \lim _{n \rightarrow \infty} \sum_{k=0}^{n} C_{n}^{k} c_{1}^{k} c_{2}^{n-k} \int_{0}^{b} u(s) d s \\
& =\lim _{n \rightarrow \infty}\left(c_{1}+c_{2}\right)^{n} \int_{0}^{b} u(s) d s \\
& =0 .
\end{aligned}
$$

Similarly, we can prove that for $n>N$ and $t \in[0, b]$,

$$
\begin{aligned}
\sum_{n=1}^{\infty} B^{n} a(t) & =\sum_{n=1}^{N} B^{n} a(t)+\sum_{n=N+1}^{\infty} B^{n} a(t) \\
& \leq \sum_{n=1}^{N} B^{n} a(t)+\sum_{n=N+1}^{\infty}\left(c_{1}+c_{2}\right)^{n} \int_{0}^{b} a(s) d s \\
& \leq \infty .
\end{aligned}
$$

Proof of (ii) is completed. 
As a consequence of (i) and (ii), we complete the proof of Theorem 3.2.

Remark 3.3 If $h(t) \equiv 0$, then (3.1) becomes

$$
u(t) \leq a(t)+\int_{0}^{t} \sum_{n=1}^{\infty} \frac{(g(t) \Gamma(\alpha))^{n}}{\Gamma(n \alpha)}(t-s)^{n \alpha-1} a(s) d s
$$

which is Theorem 1 in monograph [24].

\section{Existence result}

According to [14], we introduce the definition of the mild solution to problem (1.1).

Definition 4.1 A continuous function $x:[-\tau, b] \rightarrow X$ is a mild solution of problem (1.1) if $x(t)=\phi(t)$ on $[-\tau, 0]$ and there exists $f \in L^{1}([0, b], X)$ such that $f(t) \in F(t, x(t))$ for a.e. $t \in[0, b]$ and

$$
\begin{aligned}
x(t)= & \mathfrak{S}_{q}(t)[\phi(0)-g(0, \phi)]+g\left(t, x_{t}\right)+\int_{0}^{t}(t-s)^{q-1} A \mathfrak{T}_{q}(t-s) g\left(s, x_{s}\right) d s \\
& +\int_{0}^{t}(t-s)^{q-1} \mathfrak{T}_{q}(t-s) f(s) d s,
\end{aligned}
$$

where

$$
\begin{aligned}
& \mathfrak{S}_{q}(t)=\int_{0}^{\infty} \xi_{q}(\theta) T\left(t^{q} \theta\right) d \theta, \quad \mathfrak{T}_{q}(t)=q \int_{0}^{\infty} \theta \xi_{q}(\theta) T\left(t^{q} \theta\right) d \theta, \\
& \xi_{q}(\theta)=\frac{1}{q} \theta^{-1-\frac{1}{q}} \Psi_{q}\left(\theta^{-\frac{1}{q}}\right), \\
& \Psi_{q}(\theta)=\frac{1}{\pi} \sum_{n=1}^{\infty}(-1)^{n-1} \theta^{-q n-1} \frac{\Gamma(n q+1)}{n !} \sin (n \pi q), \quad \theta \in \mathbf{R}^{+} .
\end{aligned}
$$

Remark 4.2 ([25]) The function $\xi_{q}(\theta)$ is a probability density function defined on $\mathbf{R}^{+}$, and

$$
\int_{0}^{\infty} \theta^{v} \xi_{q}(\theta) d \theta=\int_{0}^{\infty} \frac{1}{\theta^{q v}} \Psi_{q}(\theta) d \theta=\frac{\Gamma(1+v)}{\Gamma(1+q v)}
$$

Lemma 4.3 ([14]) The operators $\mathfrak{S}_{q}$ and $\mathfrak{T}_{q}$ have the following properties:

(i) For any fixed $t \geq 0, \mathfrak{S}_{q}$ and $\mathfrak{T}_{q}$ are linear and bounded operators, that is, for any $x \in X$,

$$
\left|\mathfrak{S}_{q}(t) x\right| \leq M|x|, \quad\left|\mathfrak{T}_{q}(t) x\right| \leq \frac{q M}{\Gamma(1+q)}|x| .
$$

(ii) $\left\{\mathfrak{S}_{q}(t)\right\}_{t \geq 0}$ and $\left\{\mathfrak{T}_{q}(t)\right\}_{t \geq 0}$ are strongly continuous.

(iii) If $T(t)$ is compact for $t>0$, then $\mathfrak{S}_{q}(t)$ and $\mathfrak{T}_{q}(t)$ are also compact operators for $t>0$.

(iv) For any $x \in X, \beta \in(0,1)$, and $\eta \in(0,1]$, we have

$$
A \mathfrak{T}_{q}(t) x=A^{1-\beta} \mathfrak{T}_{q}(t) A^{\beta} x, \quad 0 \leq t \leq b,
$$


and

$$
\left\|A^{\eta} \mathfrak{T}_{q}(t)\right\| \leq \frac{q C_{\eta} \Gamma(2-\eta)}{t^{q \eta} \Gamma(1+q(1-\eta))}, \quad 0<t \leq b .
$$

Let us list the following hypotheses:

(H1) $T(t)$ is compact operator for every $t>0$.

(H2) The multivalued map $F:[0, b] \times C([-\tau, 0], X) \rightarrow \mathcal{P}_{b, c l, c v}(X)$ satisfies the following conditions:

(i) For each $t \in[0, b], F(t, \cdot)$ is upper semicontinuous, for each $x \in C([-\tau, 0], X), F(\cdot, x)$ is measurable, and the set $N_{F, x}=\left\{f \in L^{1}([0, b], X): f(t) \in F(t, x)\right.$, for a.e. $\left.t \in[0, b]\right\}$ is not empty.

(ii) For each $x \in C([-\tau, 0], X)$, there exist $m \in L^{1 / q_{1}}\left([0, b], \mathbf{R}^{+}\right)$and $r \in C\left([0, b], \mathbf{R}^{+}\right)$such that

$$
\sup \{|f(t)|: f(t) \in F(t, x)\} \leq m(t)+r(t)\|x\|_{[-\tau, 0]} \text { for a.e. } t \in[0, b]
$$

where $q_{1} \in[0, q)$.

(H3) There exists a constant $\beta \in(0,1)$ such that $g \in D\left(A^{\beta}\right), A^{\beta} g$ is continuous, and

(i) there exists a positive constant $L$ such that

$$
\left|A^{\beta} g\left(t_{1}, x_{1}\right)-A^{\beta} g\left(t_{2}, x_{2}\right)\right| \leq L\left(\left|t_{1}-t_{2}\right|+\left\|x_{1}-x_{2}\right\|_{[-\tau, 0]}\right)
$$

for $0<t_{1}, t_{2}<b, x_{1}, x_{2} \in C([-\tau, 0], X)$, and

(ii) there exist positive constants $L_{1}, L_{2}$ such that

$$
\left|A^{\beta} g(t, x)\right| \leq L_{1}\|x\|_{[-\tau, 0]}+L_{2}
$$

for any $x \in C([-\tau, 0], X)$.

Theorem 4.4 Assume that hypotheses (H1)-(H3) are satisfied. Then problem (1.1) has one mild solution, provided that

$$
L_{0}=L\left(\left\|A^{-\beta}\right\|+\frac{b^{q \beta} C_{1-\beta} \Gamma(1+\beta)}{\beta \Gamma(1+q \beta)}\right)<1, \quad\left\|A^{-\beta}\right\| L_{1}<1 .
$$

Proof Transform problem (1.1) into a fixed point problem. Consider the multivalued operator $\Phi: C([-\tau, b], X) \rightarrow \mathcal{P}(C([-\tau, b], X))$ where $\Phi x$ is defined as the set of $\rho \in C([-\tau, b], X)$ such that

$$
\rho(t)= \begin{cases}\phi(t), & t \in[-\tau, 0], \\ \mathfrak{S}_{q}(t)[\phi(0)-g(0, \phi)]+g\left(t, x_{t}\right)+\int_{0}^{t}(t-s)^{q-1} A \mathfrak{T}_{q}(t-s) g\left(s, x_{s}\right) d s & \\ \quad+\int_{0}^{t}(t-s)^{q-1} \mathfrak{T}_{q}(t-s) f(s) d s, & t \in[0, b]\end{cases}
$$

where $f \in N_{F, x}=\left\{f \in L^{1}([0, b], X): f(t) \in F\left(t, x_{t}\right)\right.$ for a.e. $\left.t \in[0, b]\right\}$. We know that the fixed point of $\Phi$ is the mild solution of problem (1.1). Consider the operators $\mathcal{A}: C([-\tau, b], X) \rightarrow$ 
$C([-\tau, b], X)$ and $\mathcal{B}: C([-\tau, b], X) \rightarrow \mathcal{P}(C([-\tau, b], X))$ defined by

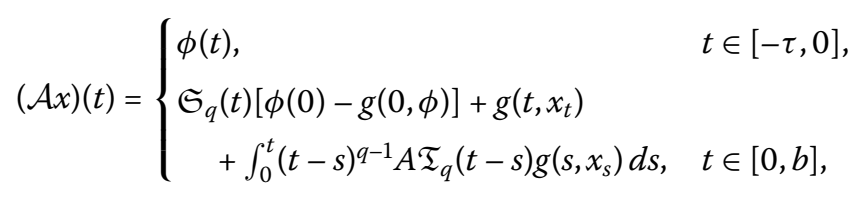

and

$$
\mathcal{B} x=\left\{\rho \in C([-\tau, b], X): \rho(t)=\left\{\begin{array}{ll}
0, & t \in[-\tau, 0], \\
\int_{0}^{t}(t-s)^{q-1} \mathfrak{T}_{q}(t-s) f(s) d s, & f \in N_{F, x}, t \in[0, b]
\end{array}\right\} .\right.
$$

It is clear that $\Phi=\mathcal{A}+\mathcal{B}$. Mild solutions of problem (1.1) are converted to the fixed points of $x \in \mathcal{A} x+\mathcal{B} x$. We shall show that the operators $\mathcal{A}$ and $\mathcal{B}$ satisfy the conditions of Theorem 2.7 by the following steps.

Step 1 . $\mathcal{A}$ is a contraction.

Let $x, y \in C([-\tau, b], X)$. Then, for each $t \in[0, b]$, from condition (H3) we have that

$$
\begin{aligned}
|(\mathcal{A} x)(t)-(\mathcal{A} y)(t)|= & \mid g\left(t, x_{t}\right)+\int_{0}^{t}(t-s)^{q-1} A \mathfrak{T}_{q}(t-s) g\left(s, x_{s}\right) d s \\
& -g\left(t, y_{t}\right)-\int_{0}^{t}(t-s)^{q-1} A \mathfrak{T}_{q}(t-s) g\left(s, y_{s}\right) d s \mid \\
\leq & \left|g\left(t, x_{t}\right)-g\left(t, y_{t}\right)\right|+\left|\int_{0}^{t}(t-s)^{q-1} A \mathfrak{T}_{q}(t-s)\left(g\left(s, x_{s}\right)-g\left(t, y_{s}\right)\right) d s\right| \\
\leq & L\left\|A^{-\beta}\right\|\left\|x_{t}-y_{t}\right\|_{[-\tau, 0]} \\
& +L \int_{0}^{t}(t-s)^{q-1}\left\|A^{1-\beta} \mathfrak{T}_{q}(t-s)\right\|\left\|x_{s}-y_{s}\right\|_{[-\tau, 0]} d s \\
\leq & L\left(\left\|A^{-\beta}\right\|+\frac{q C_{1-\beta} \Gamma(1+\beta)}{\Gamma(1+q \beta)} \int_{0}^{t}(t-s)^{q \beta-1} d s\right)_{0 \leq s \leq t} \sup _{0 \leq x_{s}-y_{s} \|_{[-\tau, 0]}} \\
\leq & L\left(\left\|A^{-\beta}\right\|+\frac{b^{q \beta} C_{1-\beta} \Gamma(1+\beta)}{\beta \Gamma(1+q \beta)}\right)\|x-y\|_{[-\tau, b]} \\
= & L_{0}\|x-y\|_{[-\tau, b]} .
\end{aligned}
$$

Therefore,

$$
\|\mathcal{A} x-\mathcal{A} y\|_{[-\tau, b]} \leq L_{0}\|x-y\|_{[-\tau, b]},
$$

so that $\mathcal{A}$ is a contraction since $L_{0}<1$.

Step 2. $\mathcal{B} x$ is convex for each $x \in C([-\tau, b], X)$.

Indeed, if $\rho_{1}$ and $\rho_{2}$ belong to $\mathcal{B} x$, then there exist $f_{1}, f_{2} \in N_{F, x}$ such that for each $t \in[0, b]$, we have

$$
\rho_{i}(t)=\int_{0}^{t}(t-s)^{q-1} \mathfrak{T}_{q}(t-s) f_{i}(s) d s, \quad i=1,2
$$


Let $0 \leq \lambda \leq 1$. Then for each $t \in[0, b]$, we have

$$
\left(\lambda \rho_{1}+(1-\lambda) \rho_{2}\right)(t)=\int_{0}^{t}(t-s)^{q-1} \mathfrak{T}_{q}(t-s)\left(\lambda f_{1}(s)+(1-\lambda) f_{2}(s)\right) d s .
$$

Since $N_{F, x}$ is convex, we have $\lambda \rho_{1}+(1-\lambda) \rho_{2} \in \mathcal{B} x$.

Step $3 . \mathcal{B}$ sends bounded sets to bounded sets in $C([-\tau, b], X)$.

It suffices to prove that there exists a constant $l>0$ such that for each $\rho \in \mathcal{B} x, x \in B_{k_{0}}=$ $\left\{x \in C([-\tau, b], X),\|x\|_{[-\tau, b]} \leq k_{0}\right\}$, we have $\|\rho\|_{[-\tau, b]} \leq l$.

Let $\rho \in \mathcal{B} x$. Then there exists $f \in N_{F, x}$ such that for $t \in[0, b]$, we have

$$
\rho(t)=\int_{0}^{t}(t-s)^{q-1} \mathfrak{T}_{q}(t-s) f(s) d s
$$

Then, for $t \in[0, b]$, we have

$$
|\rho(t)|=\left|\int_{0}^{t}(t-s)^{q-1} \mathfrak{T}_{q}(t-s) f(s) d s\right| \leq \frac{q M}{\Gamma(1+q)} \int_{0}^{t}(t-s)^{q-1}|f(s)| d s .
$$

From (H2), for $t \in[0, b]$, we have

$$
\begin{aligned}
\int_{0}^{t}(t-s)^{q-1}|f(s)| d s & \leq\left(\int_{0}^{t}(t-s)^{\frac{q-1}{1-q_{1}}} d s\right)^{1-q_{1}}\|m\|_{L^{\frac{1}{q_{1}}}[0, t]}+\bar{r} k_{0} \int_{0}^{t}(t-s)^{q-1} d s \\
& \leq \frac{M_{1}}{(1+a)^{1-q_{1}}} b^{(1+a)\left(1-q_{1}\right)}+\frac{\bar{r} k_{0} b^{q}}{q},
\end{aligned}
$$

where $a=\frac{q-1}{1-q_{1}} \in(-1,0), M_{1}=\|m\|_{L^{\frac{1}{q_{1}}}[0, b]}$, and $\bar{r}=\sup \{r(t), t \in[0, b]\}$.

Then from (4.2) and (4.3) we get that

$$
\begin{aligned}
\|\rho\|_{[-\tau, b]} & \leq\|\rho\|_{[-\tau, 0]}+\|\rho\|_{[0, b]} \\
& \leq 0+\frac{q M}{\Gamma(1+q)}\left[\frac{M_{1}}{(1+a)^{1-q_{1}}} b^{(1+a)\left(1-q_{1}\right)}+\frac{\bar{r} k_{0} b^{q}}{q}\right] \\
& :=l .
\end{aligned}
$$

Step 4. $\mathcal{B}$ maps bounded sets to equicontinuous sets of $C([-\tau, b], X)$.

Let $t_{1}, t_{2} \in[0, b], t_{1}<t_{2}$, and let $B_{k_{0}}=\left\{x \in C([-\tau, b], X),\|x\|_{[-\tau, b]} \leq k_{0}\right\}$ be a bounded set of $C([-\tau, b], X)$. For each $x \in B_{k_{0}}$ and $\rho \in \mathcal{B} x$, there exists $f \in N_{F, x}$ such that

$$
\rho(t)=\int_{0}^{t}(t-s)^{q-1} \mathfrak{T}_{q}(t-s) f(s) d s
$$

Then,

$$
\begin{aligned}
\left|\rho\left(t_{2}\right)-\rho\left(t_{1}\right)\right|= & \left|\int_{0}^{t_{2}}\left(t_{2}-s\right)^{q-1} \mathfrak{T}_{q}\left(t_{2}-s\right) f(s) d s-\int_{0}^{t_{1}}\left(t_{1}-s\right)^{q-1} \mathfrak{T}_{q}\left(t_{1}-s\right) f(s) d s\right| \\
\leq & \left|\int_{t_{1}}^{t_{2}}\left(t_{2}-s\right)^{q-1} \mathfrak{T}_{q}\left(t_{2}-s\right) f(s) d s\right| \\
& +\left|\int_{0}^{t_{1}}\left[\left(t_{2}-s\right)^{q-1}-\left(t_{1}-s\right)^{q-1}\right] \mathfrak{T}_{q}\left(t_{2}-s\right) f(s) d s\right|
\end{aligned}
$$




$$
\begin{aligned}
& \quad+\left|\int_{0}^{t_{1}}\left(t_{1}-s\right)^{q-1}\left[\mathfrak{T}_{q}\left(t_{2}-s\right)-\mathfrak{T}_{q}\left(t_{1}-s\right)\right] f(s) d s\right| \\
& =I_{1}+I_{2}+I_{3},
\end{aligned}
$$

where

$$
\begin{aligned}
& I_{1}=\left|\int_{t_{1}}^{t_{2}}\left(t_{2}-s\right)^{q-1} \mathfrak{T}_{q}\left(t_{2}-s\right) f(s) d s\right|, \\
& I_{2}=\left|\int_{0}^{t_{1}}\left[\left(t_{2}-s\right)^{q-1}-\left(t_{1}-s\right)^{q-1}\right] \mathfrak{T}_{q}\left(t_{2}-s\right) f(s) d s\right|, \\
& I_{3}=\left|\int_{0}^{t_{1}}\left(t_{1}-s\right)^{q-1}\left[\mathfrak{T}_{q}\left(t_{2}-s\right)-\mathfrak{T}_{q}\left(t_{1}-s\right)\right] f(s) d s\right| .
\end{aligned}
$$

By using a similar argument as that used in (4.3), we can conclude that

$$
\begin{aligned}
I_{1} \leq & \frac{q M}{\Gamma(1+q)}\left(\frac{M_{1}}{(1+a)^{1-q_{1}}}\left(t_{2}-t_{1}\right)^{(1+a)\left(1-q_{1}\right)}+\frac{\bar{r} k_{0}\left(t_{2}-t_{1}\right)^{q}}{q}\right), \\
I_{2} \leq & \frac{q M}{\Gamma(1+q)}\left[\left(\int_{0}^{t_{1}}\left(\left(t_{1}-s\right)^{q-1}-\left(t_{2}-s\right)^{q-1}\right)^{\frac{1}{1-q_{1}}} d s\right)^{1-q_{1}}\|m\|_{L^{\frac{1}{q_{1}}}\left[0, t_{1}\right]}\right. \\
& \left.+\bar{r} k_{0} \int_{0}^{t_{1}}\left(t_{1}-s\right)^{q-1}-\left(t_{2}-s\right)^{q-1} d s\right] \\
\leq & \frac{q M}{\Gamma(1+q)}\left[M_{1}\left(\int_{0}^{t_{1}}\left(\left(t_{1}-s\right)^{a}-\left(t_{2}-s\right)^{a}\right) d s\right)^{1-q_{1}}\right. \\
& \left.+\bar{r} k_{0}\left(\frac{\left(t_{2}-t_{1}\right)^{q}}{q}-\frac{t_{2}^{q}}{q}+\frac{t_{1}^{q}}{q}\right)\right] \\
= & \frac{q M}{\Gamma(1+q)}\left(\frac{M_{1}}{(1+a)^{1-q_{1}}}\left(t_{1}^{1+a}-t_{2}^{1+a}+\left(t_{2}-t_{1}\right)^{1+a}\right)^{1-q_{1}}+\frac{\bar{r} k_{0}}{q}\left(\left(t_{2}-t_{1}\right)^{q}-t_{2}^{q}+t_{1}^{q}\right)\right) \\
\leq & \frac{M M_{0}}{\Gamma(1+q)}\left(\frac{M_{1}}{(1+a)^{1-q_{1}}}\left(t_{2}-t_{1}\right)^{(1+a)\left(1-q_{1}\right)}+\frac{\bar{r} k_{0}}{q}\left(t_{2}-t_{1}\right)^{q}\right) .
\end{aligned}
$$

Hence, $\lim _{t_{2} \rightarrow t_{1}} I_{1}=0$ and $\lim _{t_{2} \rightarrow t_{1}} I_{2}=0$ independently of $x \in B_{k_{0}}$.

On the other hand,

$$
I_{3} \leq \int_{0}^{t_{1}}\left(t_{1}-s\right)^{q-1}\left\|\mathfrak{T}_{q}\left(t_{2}-s\right)-\mathfrak{T}_{q}\left(t_{1}-s\right)\right\||f(s)| d s
$$

Hypothesis (H1) and Lemma 4.3 imply that $\mathfrak{T}_{q}(t)$ is continuous for $t>0$. Then we get that $I_{3}$ tends to 0 independently of $x \in B_{k_{0}}$ as $t_{2} \rightarrow t_{1}$.

Consequently, $\left|\rho\left(t_{2}\right)-\rho\left(t_{1}\right)\right| \rightarrow 0$ independently of $x \in B_{k_{0}}$ as $t_{2} \rightarrow t_{1}$, which means that $\mathcal{B}\left(B_{k_{0}}\right)$ is equicontinuous.

Step 5. For each $t \in[0, b], V(t)=\left\{(\mathcal{B} x)(t), x \in B_{k_{0}}\right\}$ is relatively compact in $X$.

Obviously, $V(0)=\{0\}$ is relatively compact in $X$. Let $0<t \leq b$ be fixed. For $x \in B_{k_{0}}$ and $\rho \in \mathcal{B} x$, there exists $f \in N_{F, x}$ such that

$$
\rho(t)=\int_{0}^{t}(t-s)^{q-1} \mathfrak{T}_{q}(t-s) f(s) d s
$$


For arbitrary $\epsilon \in(0, t)$ and $\delta>0$, define the operator $\Pi_{\epsilon, \delta}$ on $B_{k_{0}}$ by

$$
\begin{aligned}
\left(\Pi_{\epsilon, \delta} x\right)(t) & =q \int_{0}^{t-\epsilon} \int_{\delta}^{\infty} \theta(t-s)^{q-1} \xi_{q}(\theta) T\left((t-s)^{q} \theta\right) f(s) d \theta d s \\
& =q \int_{0}^{t-\epsilon} \int_{\delta}^{\infty} \theta(t-s)^{q-1} \xi_{q}(\theta) T\left(\epsilon^{q} \delta\right) T\left((t-s)^{q} \theta-\epsilon^{q} \delta\right) f(s) d \theta d s \\
& =T\left(\epsilon^{q} \delta\right) q \int_{0}^{t-\epsilon} \int_{\delta}^{\infty} \theta(t-s)^{q-1} \xi_{q}(\theta) T\left((t-s)^{q} \theta-\epsilon^{q} \delta\right) f(s) d \theta d s .
\end{aligned}
$$

According to the compactness of $T(t), t>0$, we get that the set $V_{\epsilon, \delta}(t)=\left\{\left(\Pi_{\epsilon, \delta} x\right)(t), x \in B_{k_{0}}\right\}$ is relatively compact in $X$ for all $\epsilon \in(0, t)$ and $\delta>0$. Moreover, for every $x \in B_{k_{0}}$, we have

$$
\begin{aligned}
\left|(\mathcal{B} x)(t)-\left(\Pi_{\epsilon, \delta} x\right)(t)\right| & \mid q \int_{0}^{t} \int_{0}^{\delta} \theta(t-s)^{q-1} \xi_{q}(\theta) T\left((t-s)^{q} \theta\right) f(s) d \theta d s \\
& +q \int_{0}^{t} \int_{\delta}^{\infty} \theta(t-s)^{q-1} \xi_{q}(\theta) T\left((t-s)^{q} \theta\right) f(s) d \theta d s \\
& -q \int_{0}^{t-\epsilon} \int_{\delta}^{\infty} \theta(t-s)^{q-1} \xi_{q}(\theta) T\left((t-s)^{q} \theta\right) f(s) d \theta d s \mid \\
\leq & q\left|\int_{0}^{t} \int_{0}^{\delta} \theta(t-s)^{q-1} \xi_{q}(\theta) T\left((t-s)^{q} \theta\right) f(s) d \theta d s\right| \\
& +q\left|\int_{t-\epsilon}^{t} \int_{\delta}^{\infty} \theta(t-s)^{q-1} \xi_{q}(\theta) T\left((t-s)^{q} \theta\right) f(s) d \theta d s\right| \\
\leq & q M \int_{0}^{t}(t-s)^{q-1}|f(s)| d s \int_{0}^{\delta} \theta \xi_{q}(\theta) d \theta \\
& +q M \int_{t-\epsilon}^{t}(t-s)^{q-1}|f(s)| d s \int_{0}^{\infty} \theta \xi_{q}(\theta) d \theta .
\end{aligned}
$$

In view of (4.3), we have

$$
\begin{aligned}
& \left|(\mathcal{B} x)(t)-\left(\Pi_{\epsilon, \delta} x\right)(t)\right| \\
& \leq q M \int_{0}^{\delta} \theta \xi_{q}(\theta) d \theta\left(\frac{M_{1}}{(1+a)^{1-q_{1}}} b^{(1+a)\left(1-q_{1}\right)}+\frac{\bar{r} k_{0} b^{q}}{q}\right) \\
& \quad+\frac{q M}{\Gamma(1+q)}\left[\left(\int_{t-\epsilon}^{t}(t-s)^{\frac{q-1}{1-q_{1}}} d s\right)^{1-q_{1}} \| m_{L^{\frac{1}{q_{1}}}[t-\epsilon, t]}+\bar{r} k_{0} \int_{t-\epsilon}^{t}(t-s)^{q-1} d s\right] \\
& \leq q M \int_{0}^{\delta} \theta \xi_{q}(\theta) d \theta\left(\frac{M_{1}}{(1+a)^{1-q_{1}}} b^{(1+a)\left(1-q_{1}\right)}+\frac{\bar{r} k_{0} b^{q}}{q}\right) \\
& \quad+\frac{q M}{\Gamma(1+q)}\left(\frac{M_{1}}{(1+a)^{1-q_{1}}} \epsilon^{(1+a)\left(1-q_{1}\right)}+\frac{\bar{r} k_{0} \epsilon^{q}}{q}\right) .
\end{aligned}
$$

From $\lim _{\delta \rightarrow 0} \int_{0}^{\delta} \theta \xi_{q}(\theta) d \theta=0$ we conclude that there exist relatively compact sets arbitrarily approximating the sets $V(t), t>0$. Hence, the sets $V(t)$ are relatively compact in $X$ for all $t>0$. 
Step 6. $\mathcal{B}$ has a closed graph.

Let $x^{n} \rightarrow x^{*}, \rho_{n} \in \mathcal{B} x^{n}$, and $\rho_{n} \rightarrow \rho_{*}$ as $n \rightarrow \infty$. We shall prove that $\rho_{*} \in \mathcal{B} x^{*}$. Since $\rho_{n} \in \mathcal{B} x^{n}$, there exists $f_{n} \in N_{F, x^{n}}$ such that

$$
\rho_{n}(t)=\int_{0}^{t}(t-s)^{q-1} \mathfrak{T}_{q}(t-s) f_{n}(s) d s, \quad t \in[0, b] .
$$

We need to prove that there exists $f_{*} \in N_{F, x^{*}}$ such that

$$
\rho_{*}(t)=\int_{0}^{t}(t-s)^{q-1} \mathfrak{T}_{q}(t-s) f_{*}(s) d s, \quad t \in[0, b] .
$$

Consider the continuous operator $\mathcal{T}: L^{1}([0, b], X) \rightarrow C([0, b], X)$ defined by

$$
(\mathcal{T} f)(t)=\int_{0}^{t}(t-s)^{q-1} \mathfrak{T}_{q}(t-s) f(s) d s
$$

We can easily obtain that $\mathcal{T}$ is continuous. On the other hand,

$$
\left|\rho_{n}(t)-\rho_{*}(t)\right| \leq\left\|\rho_{n}-\rho_{*}\right\| \rightarrow 0 \quad \text { as } n \rightarrow \infty .
$$

From Theorem 2.6 it follows that $\mathcal{T} \circ N_{F}$ is a closed graph operator. Moreover, we have that

$$
\rho_{n} \in \mathcal{T}\left(N_{F, x^{n}}\right)
$$

Since $x_{n} \rightarrow x^{*}$, by Theorem 2.6 there exists $f_{*} \in N_{F, x^{*}}$ such that

$$
\rho_{*}(t)=\int_{0}^{t}(t-s)^{q-1} \mathfrak{T}_{q}(t-s) f_{*}(s) d s, \quad t \in[0, b] .
$$

This implies that $\rho_{*} \in \mathcal{B} x^{*}$.

Therefore, the multivalued map $\mathcal{B}$ is completely continuous and u.s.c. with convex closed values.

Step 7. The set $U=\{u \in C([-\tau, 0], X) \mid u \in \lambda \mathcal{A} u+\lambda \mathcal{B} u, 0<\lambda<1\}$ is bounded.

Let $x \in U$, then $x \in \lambda \Phi x$ for some $0<\lambda<1$. Thus, there exists $f \in N_{F, x}$ such that for $t \in[0, b]$,

$$
\begin{aligned}
x(t)= & \lambda\left(\mathfrak{S}_{q}(t)[\phi(0)-g(0, \phi)]+g\left(t, x_{t}\right)+\int_{0}^{t}(t-s)^{q-1} A \mathfrak{T}_{q}(t-s) g\left(s, x_{s}\right) d s\right. \\
& \left.+\int_{0}^{t}(t-s)^{q-1} \mathfrak{T}_{q}(t-s) f(s) d s\right) .
\end{aligned}
$$

By (H2) and (H3), for each $t \in[0, b]$, we have

$$
\begin{aligned}
|x(t)|= & \lambda \mid \mathfrak{S}_{q}(t)[\phi(0)-g(0, \phi)]+g\left(t, x_{t}\right)+\int_{0}^{t}(t-s)^{q-1} A \mathfrak{T}_{q}(t-s) g\left(s, x_{s}\right) d s \\
& +\int_{0}^{t}(t-s)^{q-1} \mathfrak{T}_{q}(t-s) f(s) d s \mid
\end{aligned}
$$




$$
\begin{aligned}
& \leq\left\|\mathfrak{S}_{q}(t)\right\||\phi(0)-g(0, \phi)|+\left\|A^{-\beta}\right\|\left|A^{\beta} g\left(t, x_{t}\right)\right| \\
& +\int_{0}^{t}(t-s)^{q-1}\left\|A^{1-\beta} \mathfrak{T}_{q}(t-s)\right\|\left|A^{\beta} g\left(s, x_{s}\right)\right| d s \\
& +\int_{0}^{t}(t-s)^{q-1}\left\|\mathfrak{T}_{q}(t-s)\right\||f(s)| d s \\
& \leq M(|\phi(0)|+|g(0, \phi)|)+\left\|A^{-\beta}\right\|\left(L_{1}\left\|x_{t}\right\|_{[-\tau, 0]}+L_{2}\right) \\
& +\int_{0}^{t}(t-s)^{q-1} \frac{q C_{1-\beta} \Gamma(1+\beta)}{(t-s)^{q(1-\beta)} \Gamma(1+q \beta)}\left(L_{1}\left\|x_{s}\right\|_{[-\tau, 0]}+L_{2}\right) d s \\
& +\frac{q M}{\Gamma(1+q)} \int_{0}^{t}(t-s)^{q-1}|f(s)| d s \\
& \leq M(|\phi(0)|+|g(0, \phi)|)+\left\|A^{-\beta}\right\|\left(L_{1}\left\|x_{t}\right\|_{[-\tau, 0]}+L_{2}\right) \\
& +\frac{q L_{1} C_{1-\beta} \Gamma(1+\beta)}{\Gamma(1+q \beta)} \int_{0}^{t}(t-s)^{q \beta-1}\left\|x_{s}\right\|_{[-\tau, 0]} d s \\
& +\frac{q L_{2} C_{1-\beta} \Gamma(1+\beta)}{\Gamma(1+q \beta)} \int_{0}^{t}(t-s)^{q \beta-1} d s \\
& +\frac{q M}{\Gamma(1+q)} \int_{0}^{t}(t-s)^{q-1} m(s) d s+\frac{q M}{\Gamma(1+q)} \int_{0}^{t}(t-s)^{q-1} r(s)\left\|x_{s}\right\|_{[-\tau, 0]} d s \\
& \leq M(|\phi(0)|+|g(0, \phi)|)+\left\|A^{-\beta}\right\|\left(L_{1}\left\|x_{t}\right\|_{[-\tau, 0]}+L_{2}\right) \\
& +\frac{q L_{1} C_{1-\beta} \Gamma(1+\beta)}{\Gamma(1+q \beta)} \int_{0}^{t}(t-s)^{q \beta-1}\left\|x_{s}\right\|_{[-\tau, 0]} d s+\frac{L_{2} C_{1-\beta} b^{q \beta} \Gamma(1+\beta)}{\beta \Gamma(1+q \beta)} \\
& +\frac{q M M_{1}}{(1+a)^{1-q_{1}} \Gamma(1+q)} b^{(1+a)\left(1-q_{1}\right)}+\frac{q M \bar{r}}{\Gamma(1+q)} \int_{0}^{t}(t-s)^{q-1}\left\|x_{s}\right\|_{[-\tau, 0]} d s .
\end{aligned}
$$

Consider the function

$$
\mu(t)=\max \{|x(s)|: s \in[-\tau, t]\}, \quad t \in[0, b] .
$$

Let $t^{*} \in[-\tau, t]$ be such that $\mu(t)=\left|x\left(t^{*}\right)\right|$. If $t^{*} \in[0, t]$, then for $t \in[0, b]$, we have

$$
\begin{aligned}
\mu(t) \leq & M(|\phi(0)|+|g(0, \phi)|)+\left\|A^{-\beta}\right\|\left(L_{1} \mu(t)+L_{2}\right) \\
& +\frac{q L_{1} C_{1-\beta} \Gamma(1+\beta)}{\Gamma(1+q \beta)} \int_{0}^{t^{*}}\left(t^{*}-s\right)^{q \beta-1} \mu(s) d s+\frac{L_{2} C_{1-\beta} b^{q \beta} \Gamma(1+\beta)}{\beta \Gamma(1+q \beta)} \\
& +\frac{q M M_{1}}{(1+a)^{1-q_{1}} \Gamma(1+q)} b^{(1+a)\left(1-q_{1}\right)}+\frac{q M \bar{r}}{\Gamma(1+q)} \int_{0}^{t^{*}}\left(t^{*}-s\right)^{q-1} \mu(s) d s .
\end{aligned}
$$

Since $\mu(t)$ is nondecreasing, for $t^{*} \in[0, t]$, we have

$$
\begin{aligned}
\int_{0}^{t^{*}}\left(t^{*}-s\right)^{q-1} \mu(s) d s & =\int_{0}^{t^{*}} s^{q-1} \mu\left(t^{*}-s\right) d s \\
& \leq \int_{0}^{t} s^{q-1} \mu(t-s) d s=\int_{0}^{t}(t-s)^{q-1} \mu(s) d s .
\end{aligned}
$$


Therefore,

$$
\begin{aligned}
\mu(t) \leq & M(|\phi(0)|+|g(0, \phi)|)+\left\|A^{-\beta}\right\|\left(L_{1} \mu(t)+L_{2}\right) \\
& +\frac{q L_{1} C_{1-\beta} \Gamma(1+\beta)}{\Gamma(1+q \beta)} \int_{0}^{t}(t-s)^{q \beta-1} \mu(s) d s+\frac{L_{2} C_{1-\beta} b^{q \beta} \Gamma(1+\beta)}{\beta \Gamma(1+q \beta)} \\
& +\frac{q M M_{1}}{(1+a)^{1-q_{1}} \Gamma(1+q)} b^{(1+a)\left(1-q_{1}\right)}+\frac{q M \bar{r}}{\Gamma(1+q)} \int_{0}^{t}(t-s)^{q-1} \mu(s) d s .
\end{aligned}
$$

If $t^{*} \in[-\tau, 0]$, then $\mu(t)=\|\phi\|_{[-\tau, 0]}$.

Therefore,

$$
\begin{aligned}
\mu(t) \leq & \|\phi\|_{[-\tau, 0]}+M(|\phi(0)|+|g(0, \phi)|)+\left\|A^{-\beta}\right\|\left(L_{1} \mu(t)+L_{2}\right) \\
& +\frac{q L_{1} C_{1-\beta} \Gamma(1+\beta)}{\Gamma(1+q \beta)} \int_{0}^{t}(t-s)^{q \beta-1} \mu(s) d s+\frac{L_{2} C_{1-\beta} b^{q \beta} \Gamma(1+\beta)}{\beta \Gamma(1+q \beta)} \\
& +\frac{q M M_{1}}{(1+a)^{1-q_{1}} \Gamma(1+q)} b^{(1+a)\left(1-q_{1}\right)}+\frac{q M \bar{r}}{\Gamma(1+q)} \int_{0}^{t}(t-s)^{q-1} \mu(s) d s .
\end{aligned}
$$

From $\left\|A^{-\beta}\right\| L_{1}<1$ we get that

$$
\begin{aligned}
\mu(t) \leq & \frac{1}{1-\left\|A^{-\beta}\right\| L_{1}}\left(\|\phi\|_{[-\tau, 0]}+M(|\phi(0)|+|g(0, \phi)|)+L_{2}\left\|A^{-\beta}\right\|\right. \\
& \left.+\frac{L_{2} C_{1-\beta} b^{q \beta} \Gamma(1+\beta)}{\beta \Gamma(1+q \beta)}+\frac{q M M_{1}}{(1+q)^{1-q_{1}} \Gamma(1+q)} b^{(1+q)\left(1-q_{1}\right)}\right) \\
& +\frac{q L_{1} C_{1-\beta} \Gamma(1+\beta)}{\left(1-\left\|A^{-\beta}\right\| L_{1}\right) \Gamma(1+q \beta)} \int_{0}^{t}(t-s)^{q \beta-1} \mu(s) d s \\
& +\frac{q M \bar{r}}{\left(1-\left\|A^{-\beta}\right\| L_{1}\right) \Gamma(1+q)} \int_{0}^{t}(t-s)^{q-1} \mu(s) d s \\
= & \bar{C}+\bar{G} \int_{0}^{t}(t-s)^{q \beta-1} u(s) d s+\bar{K} \int_{0}^{t}(t-s)^{q-1} u(s) d s,
\end{aligned}
$$

where

$$
\begin{aligned}
\bar{C}= & \frac{1}{1-\left\|A^{-\beta}\right\| L_{1}}\left(\|\phi\|_{[-\tau, 0]}+M(|\phi(0)|+|g(0, \phi)|)+L_{2}\left\|A^{-\beta}\right\|\right. \\
& \left.+\frac{L_{2} C_{1-\beta} b^{q \beta} \Gamma(1+\beta)}{\beta \Gamma(1+q \beta)}+\frac{q M M_{1}}{(1+a)^{1-q_{1}} \Gamma(1+q)} b^{(1+a)\left(1-q_{1}\right)}\right), \\
\bar{G}= & \frac{q L_{1} C_{1-\beta} \Gamma(1+\beta)}{\left(1-\left\|A^{-\beta}\right\| L_{1}\right) \Gamma(1+q \beta)}, \quad \bar{K}=\frac{q M \bar{r}}{\left(1-\left\|A^{-\beta}\right\| L_{1}\right) \Gamma(1+q)} .
\end{aligned}
$$

Then by Theorem 3.2 we have

$$
\begin{aligned}
\mu(t) & \leq \bar{C}+\int_{0}^{t} \sum_{n=1}^{\infty} \sum_{k=0}^{n} C_{n}^{k} \frac{(\bar{G} \Gamma(q \beta))^{k}(\bar{K} \Gamma(q))^{n-k}}{\Gamma(k q \beta+(n-k) q)}(t-s)^{k q \beta+(n-k) q-1} \bar{C} d s \\
& \leq \bar{C}+\bar{C} \sum_{n=1}^{\infty} \sum_{k=0}^{n} C_{n}^{k} \frac{(\bar{G} \Gamma(q \beta))^{k}(\bar{K} \Gamma(q))^{n-k} b^{k q \beta+(n-k) q}}{(k q \beta+(n-k) q) \Gamma(k q \beta+(n-k) q)} .
\end{aligned}
$$


Therefore, we obtain that

$$
\|x\|_{[-\tau, b]}=\mu(b) \leq \bar{C}+\bar{C} \sum_{n=1}^{\infty} \sum_{k=0}^{n} C_{n}^{k} \frac{(\bar{G} \Gamma(q \beta))^{k}(\bar{K} \Gamma(q))^{n-k} b^{k q \beta+(n-k) q}}{(k q \beta+(n-k) q) \Gamma(k q \beta+(n-k) q)} .
$$

This shows that $U$ is bounded.

As a result of Theorem 2.7, we obtain that $\Phi$ has a fixed point, which is the mild solution of system (1.1). This completes the proof.

\section{An example}

As an application, we consider the following fractional differential inclusions:

$$
\begin{cases}\frac{\partial q}{\partial t^{q}}\left[u(t, z)-\int_{0}^{\pi} U(z, y) u_{t}(\varsigma, y) d y\right] & \\ \in \frac{\partial^{2}}{\partial z^{2}} u(t, z)+G\left(t, u_{t}(\varsigma, z)\right), & z \in[0, \pi], t \in[0, b], \\ u(t, 0)=u(t, \pi)=0, & t \in[0, b], \\ u(\varsigma, z)=\phi(\varsigma, z), & z \in[0, \pi], \varsigma \in[-\tau, 0],\end{cases}
$$

where $0<q<1, b>0$, the function $U(z, y), z, y \in[0, \pi]$ satisfies some conditions, $G$ : $[0, b] \times C([-\tau, 0], \mathbf{R}) \rightarrow \mathcal{P}(C([-\tau, 0], \mathbf{R}))$ satisfies assumptions $(\mathrm{H} 2)$, and $u_{t}(\varsigma, z)=u(t+$ $\varsigma, z), t \in[0, b], \varsigma \in[-\tau, 0]$.

Consider $X=L^{2}([0, \pi] ; \mathbf{R})$ and the operator $A: X \rightarrow X$ defined by $A w=-w^{\prime \prime}$ with the domain

$$
D(A)=\left\{w \in X, w, w^{\prime} \text { are absolutely continuous, } w^{\prime \prime} \in X, w(0)=w(1)=0\right\} .
$$

Then $-A$ generates a strongly continuous semigroup $\{T(t)\}_{t \geq 0}$, which is compact, analytic, and self-adjoint. Furthermore, $-A$ has a discrete spectrum: the eigenvalues are $-n^{2}, n \in \mathbf{N}$, with the corresponding normalized eigenvectors $v_{n}(z)=\left(\frac{2}{\pi}\right)^{\frac{1}{2}} \sin (n z)$. We have the next three properties.

(i) For each $w \in X, T(t) w=\sum_{n=1}^{\infty} e^{-n^{2} t}\left\langle w, v_{n}\right\rangle v_{n}$. In particular, $T(\cdot)$ is uniformly stable, and $\|T(t)\| \leq e^{t}$.

(ii) For each $w \in X, A^{-\frac{1}{2}} w=\sum_{n=1}^{\infty} \frac{1}{n}\left\langle w, v_{n}\right\rangle v_{n}$, and $\left\|A^{-\frac{1}{2}}\right\|=1$.

(iii) The operator $A^{\frac{1}{2}}$ is given by

$$
A^{\frac{1}{2}} w=\sum_{n=1}^{\infty} n\left\langle w, v_{n}\right\rangle v_{n}
$$

on the space $D\left(A^{\frac{1}{2}}\right)=\left\{w(\cdot) \in X, \sum_{n=1}^{\infty} n\left\langle w, v_{n}\right\rangle v_{n} \in X\right\}$.

Then the operator $A$ satisfies (2.1), (2.2), and (H1).

Hence, system (5.1) can be reformulated as

$$
\begin{cases}D^{q}\left(x(t)-g\left(t, x_{t}\right)\right) \in-A x(t)+F\left(t, x_{t}\right), & t \in[0, b], \\ x(t)=\phi(t), & t \in[-\tau, 0],\end{cases}
$$

where $x(t)(z)=u(t, z), F\left(t, x_{t}\right)(z)=G\left(t, u_{t}(\theta, z)\right), g\left(t, x_{t}\right)(z)=\int_{0}^{\pi} U(z, y) u_{t}(\theta, y) d y$, and $\phi(t) z=\phi(t, z)$. 
The following conditions are also assumed to be true.

(i) The function $U(z, y), z, y \in[0, \pi]$ is measurable, and

$$
\int_{0}^{\pi} \int_{0}^{\pi} U^{2}(z, y) d y d z<\infty
$$

(ii) The function $\frac{\partial^{2}}{\partial z^{2}} U(z, y)$ is measurable, $U(0, y)=U(\pi, y)=0$, and

$$
\left(\int_{0}^{\pi} \int_{0}^{\pi}\left(\frac{\partial^{2}}{\partial z^{2}} U(z, y)\right)^{2}\right)^{\frac{1}{2}}<\infty
$$

From [14] we have that condition (H3) of Theorem 4.4 holds with $\beta=\frac{1}{2}$.

Take $q=\frac{1}{2}$ and assume that $F$ satisfies (H2). According to Theorem 4.4, problem (5.1) admits a mild solution, provided that (4.1) holds.

\section{Competing interests}

The authors declare that there is no conflict of interests regarding the publication of this paper.

\section{Authors' contributions}

All authors contributed equally to the writing of this paper. All authors read and approved the final manuscript.

\section{Acknowledgements}

This work was supported by Natural Science Foundation of Anhui Province (1508085MA10) and Open Project of Laboratory of Intelligent Information Processing of Suzhou University (2014YKF38).

Received: 10 October 2015 Accepted: 22 January 2016 Published online: 05 February 2016

\section{References}

1. Oldham, KB, Spanier, J: The Fractional Calculus. Academic Press, New York (1974)

2. Miller, KS, Ross, B: An Introduction to the Fractional Calculus and Differential Equations. Wiley, New York (1993)

3. Podlubny, I: Fractional Differential Equation. Academic Press, San Diego (1999)

4. Delbosco, D, Rodino, L: Existence and uniqueness for a nonlinear fractional differential equation. J. Math. Anal. Appl. 204, 609-625 (1996)

5. Aizicovici, S, Mckibben, M: Existence results for a class of abstract nonlocal Cauchy problems. Nonlinear Anal. 39 , 649-668 (2000)

6. Diethelm, K, Ford, NJ: Analysis of fractional differential equations. J. Math. Anal. Appl. 265, 229-248 (2002)

7. Eidelman, SD, Kochubei, AN: Cauchy problem for fractional diffusion equations. J. Differ. Equ. 199, $211-255$ (2004)

8. Daftardar-Gejji, $\mathrm{V}$, Jafari, $\mathrm{H}$ : Analysis of a system of nonautonomous fractional differential equations involving Caputo derivatives. J. Math. Anal. Appl. 328, 1026-1033 (2007)

9. Chang, YK, Nieto, JJ: Some new existence results for fractional differential inclusions with boundary conditions. Math. Comput. Model. 49, 605-609 (2009)

10. Agarwal, RP, Benchohra, M, Hamani, S: A survey on existence results for boundary value problems of nonlinear fractional differential equations and inclusions. Acta Appl. Math. 109, 973-1033 (2010)

11. Zhou, Y, Jiao, F, Li, J: Existence and uniqueness for fractional neutral differential equations with infinite delay. Nonlinear Anal. 71, 3249-3256 (2009)

12. Agarwal, RP, Zhou, Y, He, Y: Existence of fractional neutral functional differential equations. Comput. Math. Appl. 59, 1095-1100 (2010)

13. Dos Santos, JPC, Arjunan, MM, Cuevas, C: Existence results for fractional neutral integro-differential equations with state-dependent delay. Comput. Math. Appl. 62, 1275-1283 (2011)

14. Zhou, Y, Feng, J: Existence of mild solutions for fractional neutral evolution equations. Comput. Math. Appl. 59, 1063-1077 (2010)

15. Wang, J, Zhou, Y: Existence and controllability results for fractional semilinear differential inclusions. Nonlinear Anal. 12, 3642-3653 (2011)

16. Yan, Z: Controllability of fractional-order partial neutral functional integrodifferential inclusions with infinite delay. J. Franklin Inst. 348, 2156-2178 (2011)

17. Pazy, A: Semigroups of Linear Operators and Applications to Partial Differential Equations. Springer, New York (1983)

18. Deimling, K: Multivalued Differential Equations. de Gruyter, Berlin (1992)

19. Kisielewicz, M: Differential Inclusions and Optimal Control. Kluwer Academic, Dordrecht (1991)

20. Lasota, A, Opial, Z: An application of the Kakutani-Ky-Fan theorem in the theory of ordinary differential equations. Bull. Acad. Pol. Sci., Sér. Sci. Math. Astron. Phys. 13, 781-786 (1965)

21. Dhage, BC: Multi-valued mappings and fixed points I. Nonlinear Funct. Anal. Appl. 10, 359-378 (2005)

22. Dhage, BC: Multi-valued mappings and fixed points II. Tamkang J. Math. 37, 27-46 (2006)

23. Wang, F, Zhao, Y: A two-sided inequality of gamma function. J. Math. Res. Expo. 27, 667-670 (2007) 
24. Ye, $\mathrm{H}, \mathrm{Gao}, \mathrm{J}$, Ding, Y: A generalized Gronwall inequality and its application to a fractional differential equation. J. Math. Anal. Appl. 328, 1075-1081 (2007)

25. Mainardi, F, Paradisi, P, Gorenflo, R: Probability distributions generated by fractional diffusion equations. In: Kertesz, J, Kondor, I (eds.) Econophysics: An Emerging Science. Kluwer Academic, Dordrecht (2000)

Submit your manuscript to a SpringerOpen ${ }^{\circ}$ journal and benefit from:

- Convenient online submission

Rigorous peer review

- Immediate publication on acceptance

- Open access: articles freely available online

- High visibility within the field

- Retaining the copyright to your article 